\title{
Antioxidant effect of mogrosides against oxidative stress induced by palmitic acid in mouse insulinoma NIT-1 cells
}

\author{
Q. Xu, S.Y. Chen, L.D. Deng, L.P. Feng, L.Z. Huang and R.R. Yu \\ Department of Pharmacy, Guilin Medical University, Guilin, China
}

\begin{abstract}
Excessive oxidative stress in pancreatic $\beta$ cells, caused by glucose and fatty acids, is associated with the pathogenesis of type 2 diabetes. Mogrosides have shown antioxidant and antidiabetic activities in animal models of diabetes, but the underlying mechanisms remain unclear. This study evaluated the antioxidant effect of mogrosides on insulinoma cells under oxidative stress caused by palmitic acid, and investigated the underlying molecular mechanisms. Mouse insulinoma NIT-1 cells were cultured in medium containing $0.75 \mathrm{mM}$ palmitic acid, mimicking oxidative stress. The effects of 1 mM mogrosides were determined with the dichlorodihydrofluorescein diacetate assay for intracellular reactive oxygen species (ROS) and FITCAnnexin V/PI assay for cell apoptosis. Expression of glucose transporter-2 (GLUT2) and pyruvate kinase was determined by semi-quantitative reverse-transcription polymerase chain reaction. Palmitic acid significantly increased intracellular ROS concentration 2-fold $(\mathrm{P}<0.05)$, and decreased expression of GLUT2 (by $60 \%, \mathrm{P}<0.05$ ) and pyruvate kinase (by $80 \%, \mathrm{P}<0.05$ ) mRNAs in NIT-1 cells. Compared with palmitic acid, co-treatment with $1 \mathrm{mM}$ mogrosides for $48 \mathrm{~h}$ significantly reduced intracellular ROS concentration and restored mRNA expression levels of GLUT2 and pyruvate kinase. However, mogrosides did not reverse palmitic acid-induced apoptosis in NIT-1 cells. Our results indicate that mogrosides might exert their antioxidant effect by reducing intracellular ROS and regulating expression of genes involved in glucose metabolism. Further research is needed to achieve a better understanding of the signaling pathway involved in the antioxidant effect of mogrosides.
\end{abstract}

Key words: Mogrosides; Insulin-secreting cells; Oxidative stress damage; Apoptosis

\section{Introduction}

Mogrosides, extracted from Siraitia grosvenorii (Swingle) C. Jeffrey, belong to a common family of glycosides, and consist of a common triterpenoid aglucone (mogrol) and a number of glucose residues (1). Orally administered mogrosides exhibit antioxidant and blood glucose controlling activities in the animal model of diabetes (2). In vitro experiments using insulin-secreting cells have shown that mogrosides increased insulin secretion (3). Those studies indicate that mogrosides might act directly on pancreatic $\beta$ cells to exert an antidiabetic function. However, the underlying molecular mechanism of their antidiabetic effect is unclear.

Recently, damage to pancreatic $\beta$ cells caused by oxidative stress has been reported to be associated with the pathogenesis of clinical type 2 diabetes (4). Pancreatic $\beta$ cells have a low antioxidant content, and therefore are vulnerable to oxidative stress (5), which can result in antioxidant exhaustion caused by abnormally high levels of reactive oxygen species (ROS), e.g., byproducts of excess lipid metabolism. Serum lipid concentrations are higher in patients with metabolic syndrome, a well-established precursor of clinical type 2 diabetes.

Activation of the transcription factor forkhead protein $\mathrm{O} 1$ (FOXO1) is one of the key steps in oxidative stress-related damage to pancreatic $\beta$ cells (6). Oxidative stress drives activated FOXO1 to enter the cell nucleus, suppressing expression of genes involved in glucose metabolism, such as glucose transporter-2 (GLUT2) and pyruvate kinase (7). Through this process, pancreatic $\beta$ cells might reduce ROS production from oxidative phosphorylation and protect themselves from abnormally high glucose and lipid concentrations. If this self-protecting mechanism fails to maintain cellular ROS concentrations within endurable levels, $\beta$ cells will die from oxidative stress-related damage.

Damage to $\beta$ cells caused by oxidative stress is one of the hypotheses used to explain the progression of metabolic syndrome to clinical type 2 diabetes, and emphasizes the importance of maintaining normal levels 
of blood glucose and lipids in clinical practice. It also provides a novel strategy for the development of antidiabetic drugs. By reducing intracellular ROS, exogenous antioxidants might protect $\beta$ cells from oxidative stress damage and restore normal insulin secretion function, thus delaying the progression of type 2 diabetes. Molecules with antioxidant activity, such as $\alpha$-tocopherol (8), flavonoids from Ginkgo biloba L. (9), and mogrosides (10), have shown efficacy in controlling blood glucose levels in animal models of diabetes.

In this study, we used NIT-1 cells as a pancreatic $\beta$ cell model to investigate the effect of mogrosides on ROS scavenging and their ability to protect normal pancreatic $\beta$ cell function against damage caused by palmitic acid.

\section{Material and Methods}

\section{Culture of mouse insulinoma NIT-1 cells}

Mouse insulinoma NIT-1 cells (kindly provided by professor Leping Feng, Guilin Medical College, China) were cultured in low-glucose DMEM medium (with $5.56 \mathrm{mM}$ glucose; Hyclone, China) supplemented with $10 \%$ fetal bovine serum (Hyclone) and $1 \%$ penicillinstreptomycin mixed solution (Beyotime, China). For the following experiments, NIT-1 cells were seeded onto 6well plates at a density of $5 \times 10^{6}$ cells per well.

\section{Treatment with palmitic acid and mogrosides}

To investigate the effect of mogrosides, NIT-1 cells seeded on 6-well plates with medium containing $9.375 \mathrm{~g} / \mathrm{L}$ bovine serum albumin (AMRESCO, China) were divided into four groups: 1) The blank control group was treated with culture medium only, and 2) the palmitic acid group was treated with $0.75 \mathrm{mM}$ palmitic acid (analytical purity; Sinopharm Chemical Reagent Co., Ltd., China) in the form of sodium palmitate prepared as previously reported (11). 3) The mogroside control group was treated with $1 \mathrm{mM}$ purified mogrosides (provided as a mixture containing $50 \%$ mogroside $\mathrm{V}$, Layn Natural Ingredients Corporation, China). 4) The mogroside + palmitic acid group was treated with $1 \mathrm{mM}$ mogrosides in the presence of $0.75 \mathrm{mM}$ palmitic acid. Preliminary viability and cytotoxicity tests of $0.5,0.75,1.0,2.0$, and $4.0 \mathrm{mM}$ mogrosides and a previous study (3) had indicated that mogrosides at concentrations above $1 \mathrm{mM}$ significantly impaired growth and induced death of NIT-1 cells.

NIT-1 cells were maintained in low-glucose DMEM (5.56 mM glucose) for passaging. For treatments, highglucose DMEM (25 mM glucose) was used for cell culture. Cells were incubated in the treatment conditions described above for $48 \mathrm{~h}$, then collected for further experiments.

\section{Dichlorodihydrofluorescein diacetate (DCFH-DA) assay for intracellular ROS concentration}

To measure the intracellular ROS concentration, NIT1 cells from each group were washed with serum-free high-glucose DMEM, incubated with $1 \mu \mathrm{M}$ DCFH-DA solution (Beyotime, diluted with high-glucose DMEM) in the dark for $15 \mathrm{~min}$, separately collected in individual sample tubes using $0.5 \%$ trypsin-EDTA solution (Gibco, USA), and washed twice using Hanks' balanced salt solution (HBSS, Gibco). Cells were then suspended in HBSS and the DCF fluorescence was assayed with the FACSAria III flow cytometer (Becton Dickinson, USA).

\section{FITC-Annexin V/propidium iodide (PI) apoptosis assay}

Pancreatic $\beta$ cells from each group were individually collected in sample tubes using $0.5 \%$ trypsin-EDTA solution and suspended in HBSS. About $5 \times 10^{4}$ cells from each sample were stained using an FITC-Annexin $\mathrm{V} / \mathrm{PI}$ apoptosis staining kit (Beyotime). FITC fluorescence intensity was assayed with a FACSAria III flow cytometer. Calculation of the apoptosis rate was carried out by comparison with positive threshold levels of FITC fluorescence determined in our preliminary experiments.

\section{Semi-quantitative reverse-transcription PCR for expression levels of GLUT2 and pyruvate kinase coding genes}

The total RNA of NIT-1 cells was extracted using an Ultrapure RNA Kit (CoWin Biotech Beijing, China) and reverse-transcribed into cDNA segments using a $\mathrm{HiFi}$ MMLV cDNA Kit (CoWin). Each reaction system contained $500 \mathrm{ng}$ of total RNA. cDNA of GLUT2 and pyruvate kinase were analyzed using semi-quantitative PCR with Es Taq Mastermix (CoWin) and analyzed by $2 \%$ agarose gel electrophoresis and fluorescence gel image analysis using JS-780 Automatic Gel Imager (Shanghai Peiqing Science \& Technology, China). The primers for GLUT2 and pyruvate kinase are shown in Table 1. Relative gene expression levels were calculated from the ratio of the integrated optical density of the target to the reference gene.

\section{Data analysis}

Data are reported as means $\pm S D$. Data were analyzed using one-way ANOVA followed by a least significant difference post hoc test for comparisons among multiple groups. The significance level was set at $\mathrm{P}<0.05$.

\section{Results}

\section{Mogrosides significantly decreased intracellular ROS of NIT-1 cells}

DCF fluorescence intensities were used to compare the intracellular ROS levels of NIT-1 cells among the study groups (Figure 1). Compared to the blank control, treatment with $1 \mathrm{mM}$ mogrosides significantly reduced intracellular ROS concentration $(\mathrm{P}<0.05)$, while palmitic acid treatment increased the ROS level 2.5 -fold $(P<0.05)$. 
Table 1. Primers for semi-quantitative reverse transcript PCR assay.

\begin{tabular}{lcc}
\hline Target gene & Forward primer $\left(5^{\prime}-3^{\prime}\right)$ & Reverse primer $\left(5^{\prime}-3^{\prime}\right)$ \\
\hline $\begin{array}{l}\text { Pyruvate kinase-coding gene pklr } \\
\text { (GenBank: NM_001099779.1) }\end{array}$ & GAACTTGCCAAATGCCGA & CACGCCTTCATGGTTCTCG \\
$\begin{array}{l}\text { GLUT2-coding gene s/c2a2 } \\
\text { (GenBank: NM_031197.2) }\end{array}$ & TCAGAAGACAAGATCACCG \\
$\begin{array}{l}\text { Glyceraldehyde phosphate dehydrogenase- } \\
\text { coding gene gapdh (GenBank: NM_008084.2) }\end{array}$ & AGCCTCGTCCCGTAGACA & CACCAGTAGACTCCACGACA \\
\hline
\end{tabular}

In the mogrosides + palmitic acid group, the presence of $1 \mathrm{mM}$ mogrosides reversed the palmitic acid-induced increase of intracellular ROS $(P<0.05)$, although the ROS level was nevertheless significantly higher than that of the blank control group.

\section{Gene expressions of pyruvate kinase and GLUT2}

Compared with the blank control, mogrosides treatment showed no effect on the expression of the pyruvate kinase coding gene $p k / r$, while palmitic acid significantly reduced expression of $p k l r(\mathrm{P}<0.05$; Figure $2 \mathrm{~A})$ and of the GLUT2 encoding gene slc2a2 in NIT-1 cells $(P<0.05$; Figure $2 \mathrm{~B}$ ). These results suggested that palmitic acid might impair glucose metabolism of pancreatic $\beta$ cells by down-regulating gene expression of $p k / r$ and s/c2a2. In the presence of palmitic acid, mogrosides significantly restored gene expression of pyruvate kinase $(P<0.05$; Figure 2A) and GLUT2 ( $<<0.05$; Figure $2 \mathrm{~B})$ compared with the expression observed in the palmitic acid group, suggesting that mogrosides could reverse the palmitic acid-induced damage by regulating levels of genes involved in glucose metabolism.

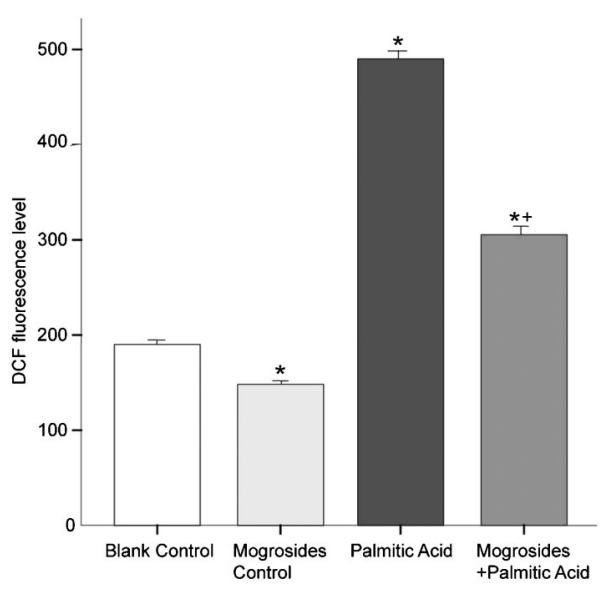

Figure 1. Comparison of intracellular reactive oxygen species (ROS) concentrations, shown as DCF fluorescence level, in different groups. Data are reported as means $\pm S D$ for $n=3$. ${ }^{*} \mathrm{P}<0.05$, compared to the blank control group; ${ }^{+} \mathrm{P}<0.05$, compared to the palmitic acid group (one-way ANOVA)

\section{Analysis of apoptosis in the NIT-1 cells}

Compared with the blank control, mogrosides showed no effect on the apoptosis of NIT-1 cells, while palmitic acid significantly increased apoptosis of NIT-1 cells $(\mathrm{P}<0.05$; Figure 3$)$. Palmitic acid-induced toxicity in pancreatic $\beta$ cells might be caused by increased intracellular ROS level in NIT-1 cells. However, in the presence of palmitic acid, mogrosides did not reverse apoptosis of NIT-1 cells ( $P=0.666$; Figure 3$)$, even though mogrosides decreased intracellular ROS levels. Extra glucose produced by mogroside hydrolysis might contribute to the increased apoptosis of NIT-1 cells.

\section{Discussion}

\section{Using an in vitro model to study oxidative stress and} pancreatic $\beta$ cell damage caused by fatty acids

Animal insulinoma cell lines, including NIT-1 cells of mouse origin, are often used as in vitro models to study the functions of pancreatic $\beta$ cells. NIT-1 cells adapt to a wide range of glucose concentrations. It has been reported that NIT-1 cells have high insulin secretion when cultured in RPMI-1640 medium containing $11.6 \mathrm{mM}$ glucose (12). In contrast, our previous experiment showed that NIT-1 cells cultured for $48 \mathrm{~h}$ in low-glucose DMEM (5.56 mM glucose), standard RPMl-1640 (11.6 mM glucose) and high-glucose DMEM (25 mM glucose) did not differ in insulin secretion. This might indicate that NIT-1 cells cultured in the standard RPMI-1640 medium for a long time lose their sensitivity to glucose, as increased glucose levels did not induce an increase in insulin secretion (13). Therefore, in this study NIT-1 cells were passaged in low-glucose DMEM to maintain their sensitivity to glucose.

Above-normal concentrations of both intracellular glucose and fat could induce glucolipotoxicity in pancreatic $\beta$ cells (14), causing more severe damage than excess glucose or fat alone. A high glucose culture environment mimics abnormally high serum glucose levels in people with impaired glucose tolerance. Compared with the NIT-1 cells cultured in $5.56 \mathrm{mM}$ glucose, cells cultured in $25 \mathrm{mM}$ glucose showed decreased gene expression of GLUT2. As expected, cells cultured in a high glucose environment displayed glucolipotoxicity in response to palmitic acid stimulation. 

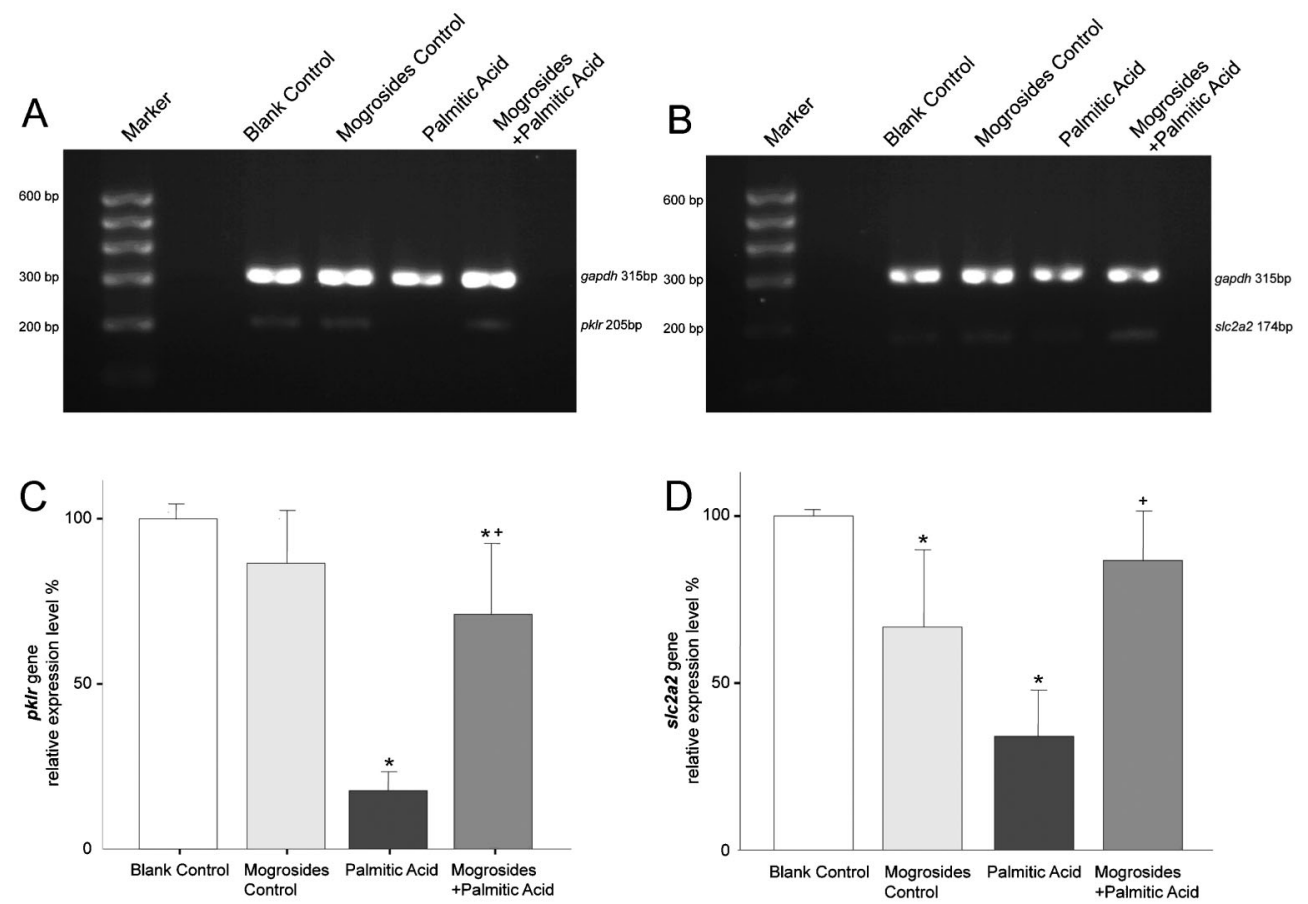

Figure 2. Electrophoresis and quantitative results of RT-PCR-amplified products on $2 \%$ agarose gel, stained with ethidium bromide and comparison of relative expression level of pyruvate kinase coding gene, $p k l r(A, C)$, and glucose tranporter-2-coding gene, s/c2a2 (B,D), in each group. gapdh: glyceraldehyde phosphate dehydrogenase-coding gene. Data are reported as means $\pm S D$ for $n=3$ per group. ${ }^{*} \mathrm{P}<0.05$, compared to the blank control group; ${ }^{+} \mathrm{P}<0.05$, compared to the palmitic acid group (one-way ANOVA).

\section{Palmitic acid and oxidative stress-caused damage in NIT-1 cells}

Palmitic acid is involved in various metabolic pathways in pancreatic $\beta$ cells. A high level of palmitic acid in the environment allows it to cross the cellular membrane and

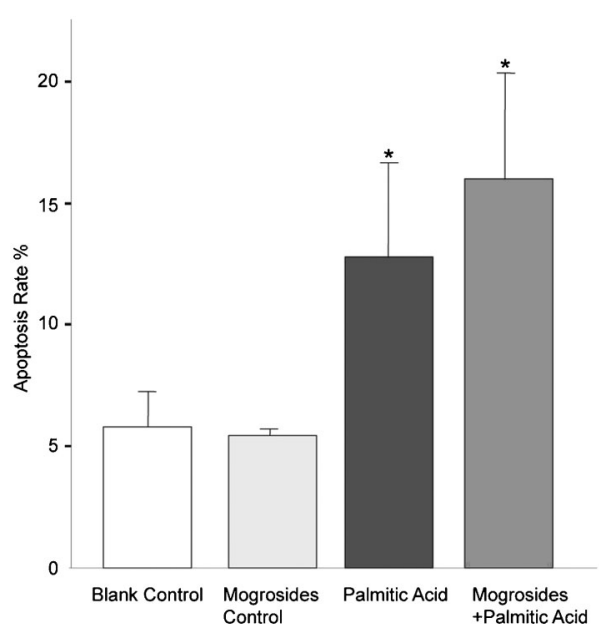

Figure 3. Comparison of apoptosis rates of NIT-1 cells in different groups. Data are reported as the means \pm SD of percentages for $n=3$ per group. ${ }^{*} P<0.05$, compared to the blank control group (one-way ANOVA). enter mitochondria where it undergoes oxidative reactions. Clearance of palmitic acid is largely dependent on metabolic reactions in which beta oxidation and oxidative phosphorylation produce enormous amounts of ROS and exhaust the supply of antioxidants in pancreatic $\beta$ cells. In pancreatic $\beta$ cells, fatty acid- or glucose-induced oxidative stress activates the c-Jun $\mathrm{NH}_{2}$-terminal kinase (JNK) signal transduction pathway and translocates an important transcription factor, FOXO1, into the cell nucleus (6). Activation of FOXO1 drives pancreatic and duodenal homeobox 1 protein (PDX1), a transcription factor initiating development and maturation of pancreatic $\beta$ cells, out of the nucleus and decreases expression of genes encoding key enzymes for glucose and fatty acid metabolism (7), resulting in decreased ROS production and oxidative stress. This might be a self-protective mechanism of pancreatic $\beta$ cells against a hostile environment containing excessive glucose and free fatty acids (14). In this study, we observed that palmitic acid treatment reduced genes of GLUT2 and pyruvate kinase by $50 \%$ in NIT-1 cells, further supporting this selfprotective mechanism. This also suggests that FOXO1 activation might be involved in palmitic acid-induced oxidative stress and mediate down-regulation of enzymes involved in glucose metabolism.

The energy-producing oxidation reaction is important for the "glucose-sensing" mechanism in pancreatic $\beta$ cells 
(15). It has been shown that palmitic acid-induced FOXO1 activation resulted in a decrease of key enzymes and transporters of glucose metabolism that impairs the glucose-induced insulin secretion (GSIS) function of pancreatic $\beta$ cells. In human studies, a single-dose infusion of palmitic acid significantly impaired the basal insulin secretion and GSIS function in healthy, obese subjects (16), implying that a similar mechanism might contribute to human chronic lipid metabolism disorders. As oxidative stress progresses, pancreatic $\beta$ cells slowly die, and proliferation of new pancreatic $\beta$ cells is inhibited by FOXO1 activation (17), causing absolute insulin deficiency.

\section{Possible mechanism of the protective effect of mogrosides on pancreatic $\boldsymbol{\beta}$ cells}

Mogroside $\mathrm{V}$ has a molecular weight of $1287 \mathrm{Da}$ and consists of a single mogrol and five glucose residues. An important question related to the protective effect of mogrosides is how these large molecules pass through the plasma membrane. In an animal pharmacokinetic study (18), mogrol and mogroside I were detected in the plasma after oral administration of a mogrosides mixture, suggesting that mogrosides might be digested by glycosidase on the surface of intestinal epithelium cells and transported into the blood stream passively. We, therefore, hypothesized that the active molecular structure of mogrosides might be mogrol, which is the main form of absorbed mogroside metabolites in animal models. As we did not directly identify the intracellular metabolites of mogrosides, we speculate that mogrosides might be hydrolyzed extracellularly, or catalyzed by glycosidase on the cell membrane. Mogrol would then cross the cell membrane passively and act in the cytoplasm as an exogenous antioxidant, scavenging ROS and lowering the oxidative stress level. In our experiment, the fact that mogroside treatment significantly lowered intracellular ROS concentration supports this hypothesis.

One molecule of mogroside $\mathrm{V}$, when fully hydrolyzed, produces five molecules of free glucose. In this study, we used $1 \mathrm{mM}$ mogrosides, which might be equivalent to $5 \mathrm{mM}$ glucose. Glucose entering pancreatic $\beta$ cells through GLUT2 is independent of insulin (19) and this is part of the "glucose-sensing" mechanism of pancreatic $\beta$ cells. Excessive glucose impairs insulin secretion and survival of pancreatic $\beta$ cells due to glucotoxicity, in which oxidative stress plays an important role. Insulinoma cell lines commonly show reduced insulin secretion and reduced survival after prolonged exposure to high concentrations of glucose (12). In this study, after $48 \mathrm{~h}$ mogroside treatment, NIT-1 showed significant reductions in expression of GLUT2 and pyruvate kinase, but no difference in apoptosis rate compared to the blank control group was observed, suggesting that mogrosides might exhibit their protective effect by delaying the onset of the adverse effects of glucose, despite the presence of more than $25 \mathrm{mM}$ glucose in the culture medium. In the presence of palmitic acid, mogrosides restored GLUT2 and pyruvate kinase expression possibly by reducing intracellular ROS, suggesting the possible involvement of oxidative stress induced by the FOXO1 activation pathway. By reducing ROS, mogrosides might inhibit the activation of signal transduction pathways and help pancreatic $\beta$ cells to survive in a high glucose or high fatty acid environment. However, we did not observe an anti-apoptosis effect of mogrosides on NIT-1 cells treated with palmitic acid. We even observed a slight trend towards a pro-apoptosis effect. This might be explained, in part, by glucolipotoxicity that is not related to oxidative stress. Although mogrosides efficiently reduced intracelIular ROS produced by metabolism of excess fatty acids and glucose, they might not prevent damage caused by metabolites produced during the process, for example advanced glycation end-products (20) or ceramine (21). These substances could induce apoptosis and other functional disorders in pancreatic $\beta$ cells.

\section{Questions for future study}

In this study, because of limited experimental resources, we only evaluated intracellular ROS concentrations and expression of genes involved in glucose metabolism. Whether activation of the JNK pathway and FOX01/PDX1 translocation into the nucleus contribute to this process remains unclear. The reason why mogrosides fail to decrease the apoptosis rate will be further explored. Dose-dependent effects of isolated mogrol and the hydrolyzed products of mogrosides also need further investigation. Mogrosides can lead to increases in superoxide dismutase and other anti-oxidant enzymes (2), but the mechanisms have not been reported. Mogrosides might affect mitochondrial glucose and fat metabolism, and change the process of oxidative phosphorylation, thus producing ROS. It has been reported that the expression of mitochondrial uncoupling protein 2 (UCP2) in pancreatic $\beta$ cells is increased under hyperlipidemia (22). Whether mogrosides affect UCP2 expression levels, thereby changing the pancreatic $\beta$ cells intracellular ROS levels, and thus improving pancreatic $\beta$ cell insulin secretion function in high glucose and high lipid environments (23), needs to be addressed in further experiments.

In addition to their antioxidant activity, a novel effect of mogrosides on pancreatic $\beta$ cells has also been reported. Recently, hydrolyzed mogrosides were reported to activate AMPK (24). The process might contribute to upregulation of genes related to glucose metabolism and restore normal insulin secretion function in pancreatic $\beta$ cells. Mogrosides might interact with intracellular receptors to achieve their effects shown in diabetes animal models. Many genes are involved in glucose metabolism (7) and lack of analysis of a large number of genes is one limitation of this study. To fully understand the anti-diabetes activity of mogrosides, all aspects 
mentioned above should be studied in depth in future research.

\section{Conclusion}

Palmitic acid increased intracellular ROS in insulinoma NIT-1 cells, while mogrosides exhibited antioxidant activity and reversed the ROS level. Mogrosides protected NIT-1 cells from palmitic acid-induced dysfunction, possibly through reducing gene expression of GLUT2 and pyruvate kinase. Mogrosides might interfere with oxidative stress-induced FOXO1 activation, which was caused by oxidants and ROS produced during metabolism of excess

\section{References}

1. Qi YP, Tang MY. Chemical composition and application research of Siraitia grosvenorii fruit (Chinese). Fujian Med J 2001; 23: 158-160.

2. Qi XY, Chen WJ, Zhang LQ, Xie BJ. Mogrosides extract from Siraitia grosvenori scavenges free radicals in vitro and lowers oxidative stress, serum glucose, and lipid levels in alloxan-induced diabetic mice. Nutr Res 2008; 28: 278-284, doi: 10.1016/j.nutres.2008.02.008.

3. Zhou Y, Zheng Y, Ebersole J, Huang CF. Insulin secretion stimulating effects of mogroside $V$ and fruit extract of luo han kuo (Siraitia grosvenori Swingle) fruit extract. Yao Xue Xue Bao 2009; 44: 1252-1257.

4. Poitout V, Robertson RP. Glucolipotoxicity: fuel excess and beta-cell dysfunction. Endocr Rev 2008; 29: 351-366, doi: 10.1210/er.2007-0023.

5. Lenzen S, Drinkgern J, Tiedge M. Low antioxidant enzyme gene expression in pancreatic islets compared with various other mouse tissues. Free Radic Biol Med 1996; 20: 463466, doi: 10.1016/0891-5849(96)02051-5.

6. Kawamori D, Kaneto $H$, Nakatani $Y$, Matsuoka TA, Matsuhisa $\mathrm{M}$, Hori $\mathrm{M}$, et al. The forkhead transcription factor Foxo1 bridges the JNK pathway and the transcription factor PDX-1 through its intracellular translocation. $J$ Biol Chem 2006; 281: 1091-1098, doi: $10.1074 / \mathrm{jbc}$. M508510200.

7. Buteau J, Shlien A, Foisy S, Accili D. Metabolic diapause in pancreatic beta-cells expressing a gain-of-function mutant of the forkhead protein Foxo1. J Biol Chem 2007; 282: 287 293, doi: 10.1074/jbc.M606118200.

8. Ihara Y, Yamada Y, Toyokuni S, Miyawaki K, Ban N, Adachi $\mathrm{T}$, et al. Antioxidant alpha-tocopherol ameliorates glycemic control of GK rats, a model of type 2 diabetes. FEBS Lett 2000; 473: 24-26, doi: 10.1016/S0014-5793(00)01489-7.

9. Wang GG, Zhang C, Li W, Zhang GB, Zhao X. Hypoglycemic effect of total flavonoids of Ginkgo biloba L. on diabetic rats (Chinese). Asia-Pacific Trad Med 2008; 4: 31-32.

10. Zhang LQ, Qi XY, Chen WJ, Song YF. Effect of mogroside extracts on blood glucose, blood lipid and antioxidation of hyperglycemic mice induced by alloxan (Chinese). Chin Pharmacol Bull 2006; 22: 237-240.

11. Cousin SP, Hugl SR, Wrede CE, Kajio H, Myers MG Jr, Rhodes CJ. Free fatty acid-induced inhibition of glucose and palmitic acid, through restoring normal glucose metabolism and insulin secretion function of pancreatic $\beta$ cells. Direct evidence of the interaction between mogrosides and signal transduction pathways is still missing. Further research is needed to achieve a better understanding of the mechanisms underlying the effect of mogrosides on pancreatic $\beta$ cells and animal models of diabetes.

\section{Acknowledgments}

Research supported by the Guangxi Natural Science Foundation of China (\#0991020).

insulin-like growth factor I-induced deoxyribonucleic acid synthesis in the pancreatic beta-cell line INS-1. Endocrinology 2001; 142: 229-240, doi: 10.1210/en. 142.1.229.

12. Luo JS, Xia N, Liang YZ. Effects of different concentrations of glucose on proliferation, secretory function and apoptosis in mouse pancreatic beta-cell line NIT-1 (Chinese). Shandong Med J 2010; 50: 28-30.

13. Li $Y$, Yang C, Wang C. Effects of culture time on insulin secretion and expression of related genes in NIT-1 cells (Chinese). Chin J Pathophysiol 2008; 24: 1811-1815.

14. Poitout V. Glucolipotoxicity of the pancreatic beta-cell: myth or reality? Biochem Soc Trans 2008; 36: 901-904, doi: 10.1042/BST0360901.

15. Pi J, Bai Y, Zhang Q, Wong V, Floering LM, Daniel K, et al. Reactive oxygen species as a signal in glucose-stimulated insulin secretion. Diabetes 2007; 56: 1783-1791, doi: 10.2337/db06-1601.

16. Xiao C, Giacca A, Carpentier A, Lewis GF. Differential effects of monounsaturated, polyunsaturated and saturated fat ingestion on glucose-stimulated insulin secretion, sensitivity and clearance in overweight and obese, non-diabetic humans. Diabetologia 2006; 49: 1371-1379, doi: 10.1007/ s00125-006-0211-x.

17. Buteau J, Spatz ML, Accili D. Transcription factor FoxO1 mediates glucagon-like peptide-1 effects on pancreatic beta-cell mass. Diabetes 2006; 55: 1190-1196, doi: $10.2337 / \mathrm{db} 05-0825$

18. Murata $Y$, Ogawa T, Suzuki YA, Yoshikawa S, Inui H, Sugiura $M$, et al. Digestion and absorption of Siraitia grosvenori triterpenoids in the rat. Biosci Biotechnol Biochem 2010; 74: 673-676, doi: 10.1271/bbb.90832.

19. Bell GI, Kayano T, Buse JB, Burant CF, Takeda J, Lin D, et al. Molecular biology of mammalian glucose transporters. Diabetes Care 1990; 13: 198-208, doi: 10.2337/diacare. 13.3.198

20. Shu TT, Zhu YX, Wang HD, Lin Y, Ma Z, Han X. AGEs decrease insulin synthesis in pancreatic beta-cell by repressing pdx-1 protein expression at the post-translational level. PLOS ONE 2011; 6: e18782, doi: 10.1371/journal. pone.0018782

21. Veluthakal R, Suresh MV, Kowluru A. Down-regulation of expression and function of nucleoside diphosphate kinase in 
insulin-secreting beta-cells under in vitro conditions of glucolipotoxicity. Mol Cell Biochem 2009; 329: 121-129, doi: 10.1007/s11010-009-0113-6.

22. Mao RR, Xue YM, Sha JP, Lin Z. Excess glucose and palmitic acid induce decoupling of respiratory chain and ATP production in mitochondria of INS-1 cells (Chinese). $J$ South Med Univ 2009; 29: 562-567.

23. Joseph JW, Koshkin V, Zhang CY, Wang J, Lowell BB,
Chan CB, et al. Uncoupling protein 2 knockout mice have enhanced insulin secretory capacity after a high-fat diet. Diabetes 2002; 51: 3211-3219, doi: 10.2337/diabetes. 51.11.3211.

24. Chen XB, Zhuang JJ, Liu JH, Lei M, Ma L, Chen J, et al. Potential AMPK activators of cucurbitane triterpenoids from Siraitia grosvenorii Swingle. Bioorg Med Chem 2011; 19: 5776-5781, doi: 10.1016/j.bmc.2011.08.030. 